\title{
Targeted treatments for multiple myeloma: specific role of carfilzomib
}

This article was published in the following Dove Press journal:

Pharmacogenomics and Personalized Medicine

20 January 2015

Number of times this article has been viewed

\author{
Dhivya Sugumar' \\ Jesse Keller ${ }^{2}$ \\ Ravi $\mathrm{Vij}^{2}$
}

'Department of Internal Medicine, St Mary's Health Center, ${ }^{2}$ Department of Medicine, Division of Oncology, Washington University in St Louis, St Louis, USA
Correspondence: Ravi Vij

Department of Medicine, Division of Oncology, Washington University in St Louis, 660 South Euclid Avenue, St Louis, MO 63II0, USA

Email rvij@dom.wustl.edu
Abstract: Carfilzomib is a selective, irreversible proteasome inhibitor, initially approved in the US in 2012 as single-agent therapy for relapsed and refractory multiple myeloma. Numerous Phase II studies have evaluated carfilzomib in the relapsed and refractory as well as the newly diagnosed setting, and Phase III studies are entering their final analysis. Data continue to grow to support its use as both single-agent therapy and in combination with immunomodulatory and other novel agents. This review discusses the role of carfilzomib in the treatment of multiple myeloma. Its mechanism of action, pharmacokinetics, and role in clinical management will be reviewed.

Keywords: relapsed and refractory, targeted therapy, proteasome inhibitor, novel agents

\section{Introduction}

Multiple myeloma (MM), a clonal plasma cell disorder, is the second-most common hematological malignancy. Incidence in the US is estimated at 24,000 people annually, with 11,000 deaths expected in 2014. ${ }^{1}$ Significant recent progress in the treatment of MM has led to clinical improvements in progression-free survival (PFS) and overall survival (OS).

While MM was once a rapidly progressive disease, initial progress was made with the introduction of melphalan and steroid combination therapy in the 1960s. The subsequent advent of autologous stem cell transplantation (ASCT) and novel agents, including the proteasome inhibitors and immunomodulatory agents, further extended OS. Continued progress is ongoing, with Surveillance Epidemiology and End Results (SEER) data showing death rates falling $1.7 \%$ per year over the past decade.

The upfront treatment of patients with novel agents has now become the standard of care for induction therapy in transplant-eligible and -ineligible patients. ${ }^{2}$ Nevertheless, responses are temporary and relapse is nearly universal. With disease progression, the majority of patients become resistant to prior lines of therapy, underlining the importance of new and effective treatments. ${ }^{3}$

A potent and highly selective proteasome inhibitor, carfilzomib is an effective and tolerable therapy for patients with MM. In 2012, it was granted fast-track approval by the US Food and Drug Administration for the treatment of MM patients who have received at least two prior therapies, including bortezomib and an immunomodulatory agent, and have demonstrated disease progression on or within 60 days of the completion of the last therapy. The data supporting its utility and safety continue to grow as further studies investigate its use in the up-front, relapsed and refractory, and combination-therapy settings. 


\section{Proteasome pathway and carfilzomib}

The ubiquitin-proteasome pathway is a crucial cellular housekeeping pathway responsible for the degradation and removal of regulatory cellular proteins. It has a central role in the regulation of cell-cycle progression and cellular senescence. Targeted proteins are enzymatically polyubiquitinated, marking them for degradation by the $26 \mathrm{~S}$ proteasome. The 26S proteasome consists of two 19S regulatory complexes, capping a central $20 \mathrm{~S}$ proteolytic core. Ubiquitinated proteins are guided into the $20 \mathrm{~S}$ core after recognition by a $19 \mathrm{~S}$ complex. Major catalytic activities of the $20 \mathrm{~S}$ core include a chymotrypsin-like (ChT-L) activity found in the $\beta 5$ subunit, a trypsin-like activity in the $\beta 2$ subunit, and a caspase-like activity in the $\beta 1$ subunit. ${ }^{4}$ ChT-L activity has been shown to be the rate-limiting step of proteolysis in vitro and in vivo. ${ }^{5}$

Carfilzomib is an irreversible, epoxyketone proteasome inhibitor that specifically targets the ChT-L activity of the $20 \mathrm{~S}$ proteasome. ${ }^{6}$ Epoxyketones are derivatives of epoxomicin, a natural product isolated from actinomycetes that forms an irreversible, selective, and highly specific adduct with the N-terminal threonine of the ChT-L $\beta 5$ subunit of the 20 S proteolytic core. ${ }^{7}$

Compared with the reversible inhibitor bortezomib, carfilzomib is a covalent inhibitor with greater in vitro selectivity and less off-target activity. ${ }^{8}$ Furthermore, it has been shown to be active in cell lines that are resistant to bortezomib and other available therapies. ${ }^{6}$ This enhanced inhibition of proteasome function leads to the accumulation of proteins targeted for degradation and subsequent cellular apoptosis. ${ }^{9} 10$

\section{Preclinical studies}

Due to the superior specificity of carfilzomib when compared to prior proteasome inhibitors, it was expected that preclinical tests would show improved proteasome inhibition and enhanced antitumor activity. Initial tests utilizing purified extracts of human $20 \mathrm{~S}$ enzyme showed carfilzomib to have a dose-dependent inhibition of ChT-L proteolytic activity. This dose-dependent inhibition persisted when investigations were pursued utilizing intact mammalian cells. ${ }^{9}$

Most cell culture-based studies evaluating cytotoxicity of prior proteasome inhibitors showed effective proapoptotic effects, but utilized long incubation times (24-72 hours) that were unrealistic mirrors of in vivo activity. Carfilzomib, however, showed impressive proteasome inhibition and cytotoxicity in hematologic tumor cell lines with short incubation times (1 hour). ${ }^{9}$
Furthermore, activity in MM cell lines showed effective induction of apoptosis in cultured tumor cells, including significant activity in bortezomib-resistant cell lines. ${ }^{11}$ Comparisons between carfilzomib and bortezomib performed via tumor xenografts in immunocompromised mouse models showed improved efficacy over bortezomib, with carfilzomib inducing significant reductions in tumor-implant burden, and minimal toxicity. ${ }^{16}$

\section{Pharmacokinetics and pharmacodynamics}

Key pharmacokinetic and pharmacodynamic studies were performed in two Phase I studies: PX-171-001 and PX-171-002. These trials found that carfilzomib is rapidly metabolized to inactive metabolites, with minimal reliance on the cytochrome P450 pathway. The mean elimination half-life was generally less than 30 minutes. Observed rates of clearance exceeded hepatic blood flow, suggesting that carfilzomib is largely cleared extrahepatically, possibly by multiple pathways. ${ }^{12-14}$

There was wide variation in both intra- and interpatient carfilzomib plasma concentrations, possibly due to differences in administration and monitoring. Both the maximum concentration and area under the curve increased with dose, but not in a dose-proportional manner. The volume of distribution at a steady state suggested wide tissue distribution in both studies. ${ }^{12-14}$

Pharmacodynamic studies evaluated inhibition of the 20S ChT-L proteasome activity in both whole blood and peripheral blood mononuclear cells (PBMCs). Samples taken 1 hour after dosing with $15 \mathrm{mg} / \mathrm{m}^{2}$ revealed more than $70 \%$ inhibition in PBMCs. At $27 \mathrm{mg} / \mathrm{m}^{2}$, approximately $90 \%$ inhibition was seen in PBMCs. In PX-171-002, which evaluated dosing on 2 consecutive days for 3 weeks of a 4-week cycle, proteasome inhibition after the second dose was equivalent to or greater than that seen with the first dose. In PX-171-001, which evaluated 5-day consecutive dosing on 2-week cycles, proteasome inhibition was observed at greater than $90 \%$ on the 5 th day of dosing. Recovery of proteasome activity was seen in PBMCs prior to the second cycle of dosing on both schedules. ${ }^{12-14}$

\section{Use in relapsed and refractory multiple myeloma}

\section{Single agent}

Approval for carfilzomib was based on the results of the Phase IIB PX-171-003-A1 trial, enrolling relapsed and refractory MM (RRMM) patients who had been previously treated 
with both bortezomib and an immunomodulatory drug. With 257 evaluable patients, this trial examined carfilzomib's efficacy in a heavily pretreated population (median of five prior therapies) that was highly bortezomib-resistant (74\%). Carfilzomib was found to have an overall response rate (ORR) of $23.7 \%$, improving to $37 \%$ when minimal responses (MRs) were included. Duration of response was 7.8 months, with median time-to-progression of 3.9 months and an OS of 15.6 months. Treatment-related grade 3-4 adverse events (AEs) were primarily fatigue (7.5\%), acute kidney injury (3.4\%), and dyspnea (3.4\%). Hematologic grade 3-4 AEs included anemia (24\%) and thrombocytopenia (29\%). ${ }^{15}$ Given its tolerable safety profile and proven efficacy, carfilzomib was granted a fast-track approval following the results of this pivotal Phase II study.

Prior to PX-171-003-A1, initial pharmacokinetics and toxicity were examined in two Phase I clinical trials: PX-171-001 and PX-171-002. The maximum tolerated dose (MTD) in PX-171-001 was $15 \mathrm{mg} / \mathrm{m}^{2}$ (carfilzomib dosed on days 1-5 every 14 days), and in PX-171-002 the MTD was not reached with patients receiving $20 \mathrm{mg} / \mathrm{m}^{2}$ in week 1 and $27 \mathrm{mg} / \mathrm{m}^{2}$ in subsequent weeks (dosing on days $1,2,8,9$, 15 , and 16 of 28-day cycles). In PX-171-001, grade 3 febrile neutropenia and grade 4 thrombocytopenia were observed, establishing the MTD at $15 \mathrm{mg} / \mathrm{m}^{2}$. PX-171-002 used an alternative dosing strategy, with grade 3-4 AEs primarily consisting of anemia, thrombocytopenia, and fatigue. Due to its improved tolerability, PX-171-002 set the standard dose regimen for further investigations. ${ }^{12,13}$

In the pivotal PX-171-003-A1 study, doses of carfilzomib were administered at $20-27 \mathrm{mg} / \mathrm{m}^{2}$, with each dose given over a period of up to 10 minutes. Building on animal data suggesting improved tolerability with prolonged infusion times, Papadopoulos et al investigated an alternative dosing strategy in a subsequent Phase I study. In this trial, 33 patients were assigned to receive carfilzomib over 30 minutes at doses escalating from $20 \mathrm{mg} / \mathrm{m}^{2}$ through $70 \mathrm{mg} / \mathrm{m}^{2}$. MTD was defined as $56 \mathrm{mg} / \mathrm{m}^{2}$, with dose-limiting toxicities of renal tubular necrosis and proteinuria (grade 3 ) in two patients at $70 \mathrm{mg} / \mathrm{m}^{2}$. The ORR was $50 \%$ for patients treated at $56 \mathrm{mg} / \mathrm{m}^{2}$ (12 patients). The addition of low-dose dexamethasone (40 mg weekly); increased the ORR to $55 \%$ among 22 patients treated at 45 or $56 \mathrm{mg} / \mathrm{m}^{2}$. Toxicities were minimal, and largely consisted of grade 1-2 anemia and thrombocytopenia. Peripheral neuropathy was rare. This confirmed the apparent safety and efficacy of a dose-escalated regimen. Investigation into optimal dosing continues. ${ }^{16}$

Further studies examined carfilzomib's role in bortezomib-naïve patients. In the Phase II PX-171-004 study, single-agent carfilzomib was evaluated in 165 patients with relapsed and/or refractory $\mathrm{MM}$ who had received one to three previous lines of therapy. A total of 129 patients with refractory, bortezomib-naïve MM were assigned to successive treatment cohorts. Initial dosing with $20 \mathrm{mg} / \mathrm{m}^{2}$ was subsequently dose-escalated to $27 \mathrm{mg} / \mathrm{m}^{2}$. The ORR was found to be $42 \%$ for patients treated with $20 \mathrm{mg} / \mathrm{m}^{2}$ over 12 cycles, and $52 \%$ for patients treated with $20 \mathrm{mg} / \mathrm{m}^{2}$ for one cycle followed by $27 \mathrm{mg} / \mathrm{m}^{2}$ for cycles 2-12. When MRs were included, the ORR increased to $59 \%$ for the lower-dose cohort and $64 \%$ for the higher-dose cohort. Time to progression was not reached in the $27 \mathrm{mg} / \mathrm{m}^{2}$ group versus 8.3 months in the $20 \mathrm{mg} / \mathrm{m}^{2}$ group. Treatment-emergent AEs of all grades included fatigue (62\%), dyspnea (38.8\%), diarrhea (31\%), anemia (41.9\%), and thrombocytopenia (30.2\%). Peripheral neuropathy was rare $(17.1 \%)$ and did not limit treatment. ${ }^{17}$

Results from pivotal Phase III studies are in the early stages of reporting data evaluating carfilzomib's efficacy. In the FOCUS trial, a randomized, controlled, Phase III trial, 315 patients with RRMM were enrolled across several international centers. Patients were randomly allocated to one of two treatment regimens: single-agent carfilzomib versus a steroid optionally combined with oral cyclophosphamide. The majority of patients allocated to steroids received cyclophosphamide. Enrolled patients had been heavily pretreated with a median of five prior lines of therapy. Preliminary reports have revealed that there was no statistically significant OS difference between the two arms. As of yet, no actual numbers for OS or PFS have been reported in the literature, and full details are pending final publication. Safety data have likewise not yet been released, but reportedly the rate of cardiac events was consistent with prior cohorts. ${ }^{18}$

\section{Combination therapy}

Given its limited off-target effects and favorable safety profile, carfilzomib is an attractive agent for use in combination treatment.

The CHAMPION-1 trial is a Phase I, dose-escalation study investigating weekly carfilzomib in combination with dexamethasone for RRMM patients. Patients were treated with carfilzomib on days 1,8 , and 15 of each 28-day cycle with an initial dose of $20 \mathrm{mg} / \mathrm{m}^{2}$ and subsequent cycles starting at $45 \mathrm{mg} / \mathrm{m}^{2}$, with dose-escalation cohorts of 56, 70, and $88 \mathrm{mg} / \mathrm{m}^{2}$. Dexamethasone was given weekly on days $1,8,15$, and 22 for the first eight cycles, after which day 22 dexamethasone was omitted. A total of 18 patients were enrolled, with two dose-limiting toxicities observed at the $88 \mathrm{mg} / \mathrm{m}^{2}$ dose level. The ORR was $67 \%$ with four complete 
responses (CR), one very good partial response (VGPR), five partial responses (PR) and three minimal responses (MR). Overall, the weekly dosing at $70 \mathrm{mg} / \mathrm{m}^{2}$ demonstrated a comparable half-life and higher area under the curve per cycle compared with the currently approved twice-weekly dosing regimen. More data are necessary to confirm this MTD and response data. Safety data were significant for two patients with grade 3 acute kidney injury, as well as one patient each with grade 3 dyspnea, pneumonia, and hyponatremia. ${ }^{19}$

Further examining the role of carfilzomib in combination with dexamethasone, Lendvai et al evaluated the efficacy of this combination in a small cohort of a Phase II study. In this trial, patients with RRMM failing to achieve at least a PR after two cycles of carfilzomib were assigned to dexamethasone in addition to carfilzomib. A total of 34 patients were treated with eleven requiring the addition of dexamethasone. Additional responses were limited, with four of the eleven patients eventually achieving a PR and continuing treatment for an average of an additional 4.5 cycles. ${ }^{20}$

The Phase IB/II PX-171-006 study examined the combination of carfilzomib, lenalidomide, and dexamethasone in patients with relapsed or progressive MM with one to three lines of previous therapy. MTD was determined for the combination in the Phase IB portion (carfilzomib $20 / 27 \mathrm{mg} / \mathrm{m}^{2}$, lenalidomide $25 \mathrm{mg}$, and dexamethasone $40 \mathrm{mg}$ ). Following a Phase II expansion, there were 84 total patients, 52 of whom were treated at the MTD. At a median of 24.4 months of follow-up, the ORR was $69 \%$ in the overall population and $76.9 \%$ in the patients treated at the MTD. Median PFS was reported at 11.8 months, with a median duration of response of 18.8 months. Grade 3-4 AEs included lymphopenia (48.1\%), neutropenia (32.7\%), fatigue (7.1\%), and diarrhea (6\%). Grade 3-4 dyspnea and peripheral neuropathy were rare, at $1.2 \%$ each. ${ }^{21,22}$

Building on the role of carfilzomib in combination with immunomodulatory agents, Shah et al evaluated the combination of carfilzomib and pomalidomide with dexamethasone (Car-Pom-d). In a Phase I/II study evaluating a heavily pretreated population of RRMM patients, the Car-Pom-d regimen was well tolerated and achieved a high response rate (ORR of $64 \%, \geq \mathrm{MR}$ rate of $81 \%$ ). Of the 72 enrolled patients, all were lenalidomide-refractory and $87 \%$ had prior bortezomib exposure. ${ }^{23}$

Additional novel combinations utilizing carfilzomib are under investigation in RRMM. A Phase I study has been presented evaluating ARRY-520, a kinesin spindle protein inhibitor, in combination with carfilzomib in patients with RRMM. ARRY-520 was administered intravenously on days
$1,2,15$, and 16 in combination with carfilzomib (days 1, 2, $8,9,15$, and 16) on 28-day cycles. Of a total of 20 patients, 19 were evaluable for outcomes. The ORR ( $\geq$ MR) was $58 \%$, with one patient having a near-CR (nCR) and six patients with a PR. Toxicity was limited and the combination was well tolerated, with six episodes of pneumonia and one episode each of febrile neutropenia and bacteremia. ${ }^{24}$

Early Phase III data are also in preliminary stages for combination therapy. The ASPIRE trial is a randomized, multicenter, open-label Phase III trial assessing the differences in PFS among relapsed MM patients enrolled onto treatment with carfilzomib in combination with lenalidomide and dexamethasone (KRd) compared to lenalidomide and dexamethasone alone. Patients were treated with carfilzomib dosed at $20 \mathrm{mg} / \mathrm{m}^{2}$ on days 1 and 2 of cycle 1 , then $27 \mathrm{mg} / \mathrm{m}^{2}$ for subsequent doses. Both arms received lenalidomide at $25 \mathrm{mg}$ per day for 21 days of a 28-day cycle in combination with dexamethasone $(\mathrm{Rd})$. Initial prepublication reports of a planned interim analysis show that with a total of 792 patients enrolled at over 100 treatment centers, the PFS for KRd was 26.3 months, a statistically significant improvement over 17.6 months for those in the $\mathrm{Rd}$ regimen. There was a trend toward improvement in OS in KRd, although this was not statistically significant at this time. Full presentation of the trial data is still pending final publication. No new concerns were raised by preliminary safety data, with similar rates of AEs as seen in previous trials. ${ }^{25}$

\section{Upfront treatment}

An ongoing area of investigation, the use of carfilzomib in the upfront setting promises to improve response rates and extend PFS and OS. Several studies have already reported the results of carfilzomib in the treatment of newly diagnosed MM.

In a Phase I/II study by Jakubowiak et al, both transplant-eligible and -ineligible newly diagnosed MM patients were evaluated. Patients on this study were enrolled onto induction therapy with carfilzomib, lenalidomide, and dexamethasone (KRd). Patients received up to eight cycles of induction therapy with carfilzomib dosed at 20,27, or $36 \mathrm{mg} / \mathrm{m}^{2}$, lenalidomide at $25 \mathrm{mg}$, and dexamethasone at $40 \mathrm{mg}$ (cycles 1-4) and $20 \mathrm{mg}$ (cycles 5-8). Transplanteligible patients were able to move to transplant (seven proceeded to transplant and 35 had stem cell collection after cycle 4). Patients could continue KRd until cycle 24. With a median follow-up of 25 months, the ORR was $98 \%$, with a CR rate of $64 \%$. At 2 years, the estimated PFS was $94 \%$ and OS was $98 \%$. Overall treatment was tolerated in patients, with a limited number of AEs. ${ }^{26}$ 
Another trial evaluating both transplant-eligible and -ineligible patients is examining the role of carfilzomib, clarithromycin, lenalidomide, and dexamethasone as combination therapy for newly diagnosed MM. Newly diagnosed patients were treated with carfilzomib (days 1, 2, 8, 9, 15, and 16 of 28-day cycles) and dexamethasone $40 \mathrm{mg}$ weekly. Treatment continued until a response plateau was achieved (no change in M protein for two cycles). Transplant-eligible patients proceeded to ASCT, and ineligible patients went straight to consolidation therapy. All patients received consolidation treatment with clarithromycin $500 \mathrm{mg}$ twice daily, lenalidomide $25 \mathrm{mg}$ daily on days $1-21$, and dexamethasone $40 \mathrm{mg}$ weekly in 28 -day cycles. Consolidation continued until a second plateau was achieved, followed by lenalidomide maintenance (10 mg daily on days 1-21 of 28-day cycles). Of a total of 23 evaluable patients, at 30.8 weeks of median follow-up, the ORR was $87 \%$, with $9 \%$ achieving a stringent $\mathrm{CR}$ (sCR), 39\% attaining a VGPR, and 35\% showing at least a PR. A total of five patients came off therapy due to grade 3 renal failure (two patients), grade 3 congestive heart failure (CHF; two patients), and a grade 3 venous thromboembolism. ${ }^{27}$

The combination of carfilzomib, lenalidomide, and dexamethasone followed by lenalidomide maintenance was investigated in newly diagnosed transplant-eligible and -ineligible MM patients. Patients were initiated onto treatment with carfilzomib $20 / 36 \mathrm{mg} / \mathrm{m}^{2}$ (days 1, 2, 8, 9, 15, and 16), lenalidomide (25 mg orally days $1-21$ ), and dexamethasone (20/10 $\mathrm{mg}$ on days $1,2,8,9,15,16,22$, and 23). Patients eligible for transplant underwent stem cell collection after cycle 4. Following eight cycles of induction, patients with at least stable disease received up to 24 cycles of lenalidomide (10 $\mathrm{mg}$ on days 1-21 of 28-day cycles). A total of 41 patients were enrolled, and after a median of nine cycles, $63 \%$ had at least a CR, $26 \%$ had a VGPR, and $8 \%$ had a PR. With a median follow-up of 10 months, PFS was $83.3 \%$. Minimal residual disease (MRD) negativity was also assessed, with $100 \%$ of the 17 patients achieving a CR also found to be negative by MRD testing. Among those attaining at least a VGPR, 63\% had evidence of abnormal plasma cells by MRD assessment. ${ }^{28}$

The CARTHADEX trial evaluated the combination of carfilzomib, thalidomide, and dexamethasone (CTD) for induction and consolidation therapy in transplant-eligible patients with newly diagnosed MM. Patients were treated with CTD for four cycles with thalidomide $200 \mathrm{mg}$ on days 1-28, dexamethasone $40 \mathrm{mg}$ on days $1,8,15$, and 22 , and carfilzomib dosed differently in three cohorts $\left(20-45 \mathrm{mg} / \mathrm{m}^{2}\right)$. Following transplantation, patients underwent consolidation with four cycles of CTD. The primary end point was VGPR after four cycles of CTD. The results of the first two carfilzomib-dose cohorts have been reported $\left(20 / 27 \mathrm{mg} / \mathrm{m}^{2}\right.$ and $36 \mathrm{mg} / \mathrm{m}^{2}$ ). With a total of 70 patients included, and median follow-up of 22 and 7 months, the ORR was $96 \%$. After induction therapy, $19 \%$ of patients achieved a CR or $\mathrm{sCR}$, and $60 \%$ had at least a VGPR. The $\mathrm{CR} / \mathrm{sCR}$ rate improved to $30 \%$ after ASCT and to $49 \%$ after consolidation therapy. The PFS was $74 \%$ in the evaluated population, with median follow-up as noted earlier. The regimen was well tolerated, with grade 3-4 nonhematologic toxicity observed in $<5 \%$ of patients, primarily infection and rash. ${ }^{29}$

The CYCLONE trial is a Phase I/II study evaluating carfilzomib combination therapy in newly diagnosed transplant-eligible MM patients. Enrolled patients are assigned to induction therapy with a combination of carfilzomib (15-45 mg/m $\mathrm{m}^{2}$ on days $1,2,8,9,15$, and 16 ), thalidomide (100 $\mathrm{mg}$ on days 1-28), cyclophosphamide (300 $\mathrm{mg} / \mathrm{m}^{2}$ on days 1,8 , and 15 ), and dexamethasone (40 $\mathrm{mg}$ weekly). As of the most recent publication, with a total of 54 patients, the ORR was $91 \%$, with $18 \%$ achieving a CR and 58\% a VGPR. Minimal toxicities were reported, with primarily grade 1 neuropathy and minimal cardiac or pulmonary effects. With a median follow-up of 16 months, 1 -year PFS was $90 \%$ and 1 -year OS was $98 \% .^{30}$

\section{Role in special populations Asymptomatic patients}

Given MM's easily observable precursor state, the role of early treatment for patients nearing a diagnosis of MM has been an ongoing clinical question. For these asymptomatic or "smoldering" MM patients, there have been multiple trials evaluating the efficacy of intervention. This remains an area of ongoing clinical investigation without clear evidence to guide clinical practice.

Investigating the role of carfilzomib in the asymptomatic patient, Landgren et al undertook a single-arm Phase II trial for patients with high-risk smoldering MM. Patients were treated with carfilzomib in combination with lenalidomide and dexamethasone, followed by 2 years of lenalidomide maintenance therapy. High-risk smoldering MM was defined by the PETHEMA or Mayo criteria. Carfilzomib was given in 28-day cycles at $20 / 36 \mathrm{mg} / \mathrm{m}^{2}$ on days $1,2,8,9,15,16,22$, and 23. After eight cycles of induction, patients with a response consistent with stable disease or better received lenalidomide maintenance $(10 \mathrm{mg}$ daily on days 1-21) for 2 years. Twelve patients were enrolled, with an ORR of $100 \%$ (five sCRs, two CRs, two 
nCRs, and three VGPRs), with no patients progressing to symptomatic MM during the study. Evaluation of MRD showed six of seven patients achieving at least a CR to be negative by MRD assessment. The most common toxicities were rash (17\%), CHF (8\%), dyspnea (8\%), and lymphopenia (33\%). Larger clinical studies and further follow-up are needed, but these results show impressive response in the smoldering MM setting. ${ }^{31}$

\section{Renal impairment}

Often a defining characteristic, renal dysfunction is a common feature of MM. Its development has been associated with worse prognosis and shortened survival, limiting the available treatment options and often requiring dose reductions. ${ }^{32}$

To assess the safety and efficacy of carfilzomib in patients with renal impairment, the Phase II PX-171005 trial enrolled relapsed/refractory or progressive MM patients who had been treated with at least two prior regimens. A total of 50 patients were enrolled and stratified by creatinine clearance $(>80 \mathrm{~mL} / \mathrm{min}, 50-80 \mathrm{~mL} / \mathrm{min}$, 30-49 $\mathrm{mL} / \mathrm{min},<30 \mathrm{~mL} / \mathrm{min}$ and chronic hemodialysis). A total of 33 of these patients $(66 \%)$ were refractory to prior bortezomib therapy. Carfilzomib was given on days $1,2,8,9,15$, and 16 in 28-day cycles, with the following dose escalation: $15 \mathrm{mg} / \mathrm{m}^{2}$ (cycle 1 ), $20 \mathrm{mg} / \mathrm{m}^{2}$ (cycle 2 ), and $27 \mathrm{mg} / \mathrm{m}^{2}$ (cycle $3+$ ). The primary goal was the assessment of pharmacokinetics, with secondary outcomes including safety, tolerability, and efficacy. ${ }^{33}$

In the final analysis, there were no differences in carfilzomib clearance or exposure among patients with impaired renal function versus those with normal renal function. Grade $3 / 4$ AEs were limited to anemia (28\%), thrombocytopenia (20\%), lymphopenia (18\%), and fatigue (14\%), and were similar among groups. The ORR was $25.5 \%$, with a median duration of response of 7.9 months. ${ }^{33}$

There appears to be no differences in carfilzomib clearance or exposure among patients with normal renal function and those with renal impairment. Additionally, the safety of carfilzomib was not influenced by the degree of baseline renal impairment, with carfilzomib being well tolerated and showing promising efficacy in all groups. ${ }^{33}$

\section{Elderly and transplant-ineligible}

Age is the greatest risk factor for the development of MM, with the disease incidence increasing steadily to a peak of more than 40 per 100,000 people in those older than 80 years. ${ }^{34}$ The accumulation of additional comorbidities with advancing age makes this subpopulation an important area for active research to improve outcomes. The initial trials of single-agent carfilzomib enrolled patients with a median age of 63.5 years (PX-171-003-AO), 63 years (PX-171-003-A1), and 65 years (PX-171-004), suggesting its efficacy and tolerability in an older population. ${ }^{15,17,35}$

Combination therapies including carfilzomib have shown encouraging tolerability and activity in patients $\geq 65$ years of age. A Phase II study by Bringhen et al evaluated the efficacy and safety of the combination of carfilzomib, cyclophosphamide, and dexamethasone in elderly patients ( $\geq 65$ years of age) or those ineligible for ASCT. A total of 58 patients were enrolled and received this combination for up to nine cycles, followed by maintenance single-agent carfilzomib. Maintenance continued until progression or intolerance. Following a median of nine cycles of induction (range 1-9), a total of $20 \%$ of patients achieved an sCR and 49\% had attained an nCR. The ORR was excellent, with $95 \%$ of patients with at least a PR and $71 \%$ with a VGPR. With a median follow-up of 18 months, the 2-year PFS was $76 \%$ and the OS rate was $87 \%$. The regimen had an acceptable safety profile in this population of older, transplant-ineligible patients. The most frequent reported toxicity was fever (32\%), with peripheral neuropathy being rare $(8 \%)$ and relatively mild (grade 1-2). ${ }^{36}$

A separate Phase I/II study by Touzeau et al examined the role of carfilzomib plus melphalan and prednisone in elderly patients with newly diagnosed MM. A total of 24 patients $>65$ years of age were enrolled in the Phase I trial and treated with carfilzomib $\left(20 \mathrm{mg} / \mathrm{m}^{2}\right.$ with escalation to $45 \mathrm{mg} / \mathrm{m}^{2}$ ), melphalan $9 \mathrm{mg} / \mathrm{m}^{2}$, and prednisone $60 \mathrm{mg} / \mathrm{m}^{2}$. MTD was $36 \mathrm{mg} / \mathrm{m}^{2}$, and the subsequent Phase II trial enrolled 45 additional patients for a total of 69 patients. ORR was $89 \%$, and at a median follow-up of 12 months, projected 2 -year OS was $89.9 \%$. Carfilzomib plus melphalan and prednisone was well tolerated without significant peripheral neuropathy, and dosing at $40 \mathrm{mg} / \mathrm{m}^{2}$ was limited by grade 3-4 fever and hypotension. ${ }^{37}$

In a subgroup analysis of a Phase I/II study by Jakubowiak et al, the efficacy of KRd was evaluated in elderly ( $\geq 65$ years of age) newly diagnosed MM patients. A total of 23 patients above the age of 65 years were enrolled. Patients were treated with KRd induction for eight cycles, followed by KRd maintenance for cycles 9-24 and then lenalidomide maintenance until progression or toxicity. The ORR was $100 \%$, with the 17 patients completing at least eight cycles of treatment, all achieving at least an nCR and 76\% reaching an sCR. One- and 2-year PFS rates were $97 \%$ and $92 \%$, respectively. ${ }^{26}$ 


\section{High-risk cytogenetics}

The role of cytogenetics in risk-stratifying prognosis for MM patients is well documented. ${ }^{38,39}$ Chromosomal alterations are well described, and are often complex in nature. ${ }^{40}$ These chromosomal aberrations have varied effects on outcomes for patients with MM. ${ }^{41}$

With the introduction of the proteasome inhibitor bortezomib, evidence of its ability to overcome the adverse impact of some cytogenetic markers was noted. ${ }^{42-44}$ To evaluate the efficacy of carfilzomib in the management of patients with adverse cytogenetics, a prospective subgroup analysis of the Phase II PX-171-003-A1 trial was performed. In this study of 257 evaluable RRMM patients, cytogenetic profiles were available for 229 patients, of whom 62 (27.1\%) had high-risk cytogenetics (defined as del17p13, t[4;14], t[14;16], or del13). The remaining 167 (72.9\%) had standard-risk cytogenetic profiles, with similar baseline characteristics among the two arms. The ORR was comparable between the groups $(25.8 \%$ versus $24.6 \%$ for high-risk and standard-risk, respectively), with a trend of shorter response duration for high-risk patients (median duration of response of 5.6 months versus 8.3 months). OS was significantly prolonged in the standardrisk group, with the average patient living 9.3 months with high-risk cytogenetics and 19.0 months for standard-risk patients. These data, particularly the equivalent response rates, supported the efficacy of carfilzomib in overcoming some of the impact of high-risk cytogenetic abnormalities, but continued to show their negative effect on OS. ${ }^{45}$

\section{Adverse effects}

Given its high specificity and irreversible binding, the side-effect profile of carfilzomib differs significantly from bortezomib. A multitrial analysis was performed to assess the safety profile of single-agent carfilzomib in 526 patients with relapsed and/or refractory MM evaluating the four main Phase II trials assessing its efficacy (PX-171-003-A0 and -A1, PX-171-004, and PX-171-005). ${ }^{49}$ In these studies, carfilzomib was administered on days 1, 2, 8, 9, 15 and 16 in 28-day cycles, with the maximum dose administered being $27 \mathrm{mg} / \mathrm{m}^{2} .^{15}$

The majority of patients evaluated in these trials were heavily pretreated with a median of four prior regimens. The most common grade 3-4 AEs were fatigue (55.5\%), anemia (46.8\%), and nausea (44.9\%). By organ system, AEs were most common in the respiratory (69\%), renal (33.1\%), and cardiac (22.1\%) systems. Hematologic toxicities included thrombocytopenia (23.4\%), anemia (22.4\%), and lymphopenia (18.1\%). Febrile neutropenia was rare, occurring in $1.1 \%$ of patients. In comparison to bortezomib, peripheral neuropathy was uncommon (13.9\%), with no grade 4 or above reported, and all grade 3 peripheral neuropathy occurred in those with baseline grade 1 or 2 neuropathy. ${ }^{46}$

Cardiac toxicity has been a concern with carfilzomib since early studies. In the aggregate analysis, 38 patients (7.2\%) developed cardiac failure events (CHF, pulmonary edema, or decreased ejection fraction). There was no difference in mortality for patients who had baseline cardiac risk factors, and the rate of cardiac events did not increase with subsequent cycles. Any cardiac AE was seen in $22.1 \%$ of patients, with the most common being: hypertension (14.3\%), arrhythmia (13.3\%), and cardiomyopathy (1.7\%). Complicating the evaluation of cardiac toxicity is the nature of MM patients, who often have multiple comorbidities and cumulative toxicities from prior therapies. In the evaluated trials, $73.6 \%$ of patients had a history of prior cardiac events, with $70 \%$ having baseline cardiac risk factors. ${ }^{46}$

Accompanying cardiac events, respiratory AEs were the most common nonhematologic organ system affected. Dyspnea was reported in $42.2 \%$ of patients; however, most episodes were grade 1-2, transient, and resolved without altering dosing. Grade 3 episodes of dyspnea occurred in only $4.8 \%$ of patients, and no grade 4 events were seen. ${ }^{46}$ Hydration has been previously recommended with carfilzomib treatment, due to concerns of acute kidney injury, and it is suspected that some episodes of dyspnea were related to aggressive fluid administration in the setting of significant cardiac comorbidities.

\section{Key ongoing studies}

As described earlier, many studies have investigated the role of carfilzomib in the RRMM setting, as well as in the newly diagnosed patient (Table 1). Ongoing Phase I, II, and III studies are evaluating carfilzomib as a combination agent in the treatment of RRMM, as well as newly diagnosed disease (Tables 2 and 3). Some of these trials have been reported and were discussed earlier, but many are actively enrolling and results are pending.

It seems likely that carfilzomib's indication will grow, with the role of combination therapies expanding and its utility in the front-line setting evolving. Ongoing comparative Phase III trials will likely further clarify carfilzomib's potential benefits.

The currently enrolling ENDEAVOR trial ${ }^{47}$ is a randomized, multicenter, open-label Phase III study of carfilzomib and dexamethasone versus bortezomib plus dexamethasone for relapsed MM patients with no more than three prior 
Table I Key completed studies evaluating carfilzomib in MM

\begin{tabular}{|c|c|c|c|c|c|c|}
\hline Study & Population & Patients & ORR (\%) & CR + VGPR & $\begin{array}{l}\text { PFS } \\
\text { (months) }\end{array}$ & $\begin{array}{l}\text { OS } \\
\text { (months) }\end{array}$ \\
\hline $\begin{array}{l}\text { PX-I7I-00I (Phase I) } \\
\text { carfilzomib (single agent) }\end{array}$ & $\begin{array}{l}\text { Hematologic malignancies } \\
\text { (MM, NHL, WM, HL) }\end{array}$ & 29 & NR & NR & NR & NR \\
\hline $\begin{array}{l}\text { PX-I7I-002 (Phase I) } \\
\text { carfilzomib (single agent) }^{12}\end{array}$ & $\begin{array}{l}\text { Hematologic malignancies } \\
\text { (MM, NHL, HL) }\end{array}$ & 48 & NR & NR & NR & NR \\
\hline $\begin{array}{l}\text { PX-I7I-003-A0 (Phase IIB) } \\
\text { carfilzomib (single agent) }\end{array}$ & RRMM & 46 & 16.7 & $0(0+0)$ & 3.5 & NR \\
\hline $\begin{array}{l}\text { PX-I7I-003-AI (Phase II) } \\
\text { carfilzomib (single agent) }\end{array}$ & RRMM & 266 & 23.7 & $5.5 \%(0.4 \%+5.1 \%)$ & 3.7 & 15.6 \\
\hline $\begin{array}{l}\text { PX-I7I-004 (Phase II) } \\
\text { carfilzomib (single agent, } \\
\left.20 \mathrm{mg} / \mathrm{m}^{2} \text { versus } 27 \mathrm{mg} / \mathrm{m}^{2}\right)^{17}\end{array}$ & RRMM & 129 & $\begin{array}{l}42.4 \\
\text { versus } 52.2\end{array}$ & $\begin{array}{l}17 \%(3.4 \%+13.6 \%) \\
\text { versus } 28.4 \%(1.5 \%+26.9 \%)\end{array}$ & $\begin{array}{l}8.2 \\
\text { versus NR }\end{array}$ & NR \\
\hline $\begin{array}{l}\text { PX-I7I-005 (Phase II) } \\
\text { carfilzomib (single agent) }^{33}\end{array}$ & $\begin{array}{l}\text { RRMM: renal } \\
\text { dysfunction }\end{array}$ & 50 & 25.5 & $0(0+0)$ & NR & NR \\
\hline $\begin{array}{l}\text { PX-I7I-006 (Phase IB) } \\
\text { carfilzomib }+\mathrm{Rd}^{22}\end{array}$ & $\begin{array}{l}\text { Relapsed or } \\
\text { progressive MM }\end{array}$ & 40 & 62.5 & $35 \%(2.5 \%+32.5 \%)$ & 10.2 & NR \\
\hline $\begin{array}{l}\text { PX-17I-006 (Phase II) } \\
\text { carfilzomib }+R^{21}\end{array}$ & $\begin{array}{l}\text { Relapsed or } \\
\text { progressive MM }\end{array}$ & 52 & 76.9 & $42.2 \%(5.7 \%+36.5 \%)$ & 28.7 & 37 \\
\hline $\begin{array}{l}\text { PX-I7I-007 (Phase IB/II) } \\
\text { carfilzomib (single agent) } \\
\text { dose-expansion cohort }{ }^{16}\end{array}$ & $\begin{array}{l}\text { Relapsed MM, solid } \\
\text { tumors or lymphoma }\end{array}$ & 33 & 55 & $28 \%(5 \%+23 \%)$ & 7 & NR \\
\hline Carfilzomib + Rd (Phase I/II) ${ }^{26}$ & Newly diagnosed & 53 & 98 & $81 \%(62 \%+19 \%)$ & NR & NR \\
\hline $\begin{array}{l}\text { Carfilzomib + clarithromycin + } \\
\text { Rd (Phase I) })^{27}\end{array}$ & Newly diagnosed & 24 & 87 & $61 \%(13 \%+48 \%)$ & NR & NR \\
\hline $\begin{array}{l}\text { FOCUS: PX-I7I-0II (Phase III) } \\
\text { carfilzomib versus best } \\
\text { supportive care }{ }^{18}\end{array}$ & RRMM & 315 & NR & NR & NR & NR \\
\hline $\begin{array}{l}\text { ASPIRE: PX-I7I-009 (Phase III) } \\
\text { carfilzomib + lenalidomide + } \\
\text { dexamethasone versus } \\
\text { lenalidomide + dexamethasone } \\
\text { Planned interim analysis }{ }^{25}\end{array}$ & Relapsed MM & 792 & NR & NR & $\begin{array}{l}26.3 \\
\text { versus } 17.6\end{array}$ & NR \\
\hline
\end{tabular}

therapies. Patients must have had at least one prior therapy to which a response was recorded. Given carfilzomib's improved safety profile and increased specificity, this will compare the outcomes and safety of bortezomib versus carfilzomib in relapsed MM patients. The primary outcome is PFS.

The CLARION trial is a Phase III study of carfilzomib, melphalan, and prednisone versus bortezomib, melphalan, and prednisone in newly diagnosed MM patients who are ineligible for transplant. The primary outcome is PFS, with efficacy and safety as key secondary outcomes. ${ }^{48}$

\section{Conclusion}

Significant changes in the management of MM over the past several decades have greatly improved outcomes. Despite many advances, MM remains an incurable disease. The

Table 2 Key ongoing trials in newly diagnosed multiple myeloma (MM)

\begin{tabular}{|c|c|c|c|c|}
\hline Regimen & Population & Authors & Patients & Phase \\
\hline Carfilzomib + bendamustine + dexamethasone & Newly diagnosed & Lentzsch et $\mathrm{al}^{49}$ & 34 & $\mathrm{I} / \mathrm{IIB}$ \\
\hline Carfilzomib + high-dose melphalan & Transplant-eligible (preparative regimen) & Costa et $\mathrm{al}^{50}$ & 58 & $\mathrm{I} / \mathrm{IIA}$ \\
\hline $\begin{array}{l}\text { Carfilzomib + melphalan + prednisone versus } \\
\text { bortezomib + melphalan }+ \text { prednisone }(\text { CLARION })\end{array}$ & Transplant-ineligible, newly diagnosed & Facon et $\mathrm{al}^{48}$ & 882 & III \\
\hline $\begin{array}{l}\text { Initial and posttransplant treatment with } \\
\text { carfilzomib + revlimid + dexamethasone }\end{array}$ & $\begin{array}{l}\text { Newly diagnosed, transplant-eligible, } \\
\text { pre- and post-ASCT }\end{array}$ & Jakubowiak et $\mathrm{al}^{51}$ & 44 & II \\
\hline Carfilzomib + lenalidomide + dexamethasone & Smoldering MM (high risk) & Landgren et $\mathrm{al}^{52}$ & 30 & II \\
\hline
\end{tabular}

Abbreviation: ASCT, autologous stem cell transplantation. 
Table 3 Key ongoing trials in relapsed or refractory multiple myeloma

\begin{tabular}{|c|c|c|c|c|}
\hline Regimen & Population & Author(s) & Patients & Phase \\
\hline $\begin{array}{l}\text { High-dose carfilzomib for MM patients with } \\
\text { progression on standard dose carfilzomib }\end{array}$ & RRMM & Chari et $\mathrm{a}^{53}$ & 36 & II \\
\hline Bruton's tyrosine-kinase inhibitor + carfilzomib in RRMM & RRMM & Pharmacyclics $^{54}$ & 223 & $\mathrm{I} / \mathrm{IB}$ \\
\hline Arry-520 + carfilzomib & RRMM & Shah et $\mathrm{al}^{55}$ & 76 & $\mathrm{I} / \mathrm{II}$ \\
\hline $\begin{array}{l}\text { Two dose levels of carfilzomib + dexamethasone } \\
\text { for RRMM (SI304) }\end{array}$ & RRMM & Ailawadhi et a ${ }^{56}$ & 140 & II \\
\hline Panobinostat + carfilzomib & RRMM & Kaufman et $\mathrm{al}^{57}$ & 36 & I \\
\hline Pomalidomide + dexamethasone + carfilzomib $(\mathrm{PdC})$ & RRMM & Jakubowiak et al ${ }^{58}$ & 92 & $\mathrm{IB} / \mathrm{II}$ \\
\hline Carfilzomib + lenalidomide + vorinostat + dexamethasone & RRMM & Siegel et al ${ }^{59}$ & 50 & $\mathrm{I} / \mathrm{II}$ \\
\hline $\begin{array}{l}\text { Carfilzomib + pegylated liposomal doxorubicin }+ \\
\text { dexamethasone }\end{array}$ & RRMM & $V_{i j}$ et al ${ }^{60}$ & 53 & $\mathrm{I} / \mathrm{II}$ \\
\hline 60-Minute infusion of carfilzomib & RRMM & Berenson et $a^{\mid 61}$ & 38 & $\mathrm{I} / \mathrm{II}$ \\
\hline $\begin{array}{l}\text { Carfilzomib/SAHA + high-dose gemcitabine/busulfan/ } \\
\text { melphalan + ASCT }\end{array}$ & RRMM & Nieto ${ }^{62}$ & 75 & II \\
\hline
\end{tabular}

Abbreviations: RRMM, relapsed and refractory multiple myeloma; MM, multiple myeloma; SAHA, suberoylanilide hydroxamic acid; ASCT, autologous stem cell transplantation.

development of new drugs and novel combinations is key to continued progress.

Current data show that carfilzomib is an active and tolerable agent in the treatment of RRMM. Evidence for its efficacy and safety continue to grow. Its role in the bortezomib-refractory patient was clearly illustrated in the PX-171-003-A1 trial, making it a viable and effective option for patients who were previously refractory to proteasome inhibition.

While numerous Phase II trials have shown it to have excellent response rates and a tolerable safety profile, initial Phase III studies are just now being reported. While aggregate results are encouraging, there continues to be much to learn about carfilzomib's role in the management of the newly diagnosed patient, as well as its efficacy and tolerability as combination therapy. Further studies will continue to clarify the optimal dosing regimen and technique to maximize safety and efficacy.

The role of carfilzomib in the setting of emerging oral proteasome inhibitors remains to be seen. While patient preferences may strongly favor oral options, their comparative efficacy and side-effect profiles are still under investigation. It is likely there will continue to be a clear role for parenteral proteasome inhibitors despite the advent of oral options. Further questions yet to be answered include the role of carfilzomib in the maintenance setting and the variable utility of proteasome inhibitors in the era of personalized medicine.

Overall, carfilzomib represents an excellent option for patients with MM. We expect its indication to grow and its regulatory approval to expand outside the US. As data continue to accumulate supporting its efficacy and safety, it will maintain its role as a backbone of treatment.

\section{Disclosure}

The authors report no conflicts of interest in this work.

\section{References}

1. American Cancer Society. Multiple Myeloma. August 10, 2014]; Available from: http://www.cancer.org/cancer/multiplemyeloma/.

2. National Comprehensive Cancer Network. Multiple Myeloma (Version 1.2015). August 10, 2014 Available from: http://www.nccn.org/ professionals/physician_gls/pdf/myeloma.pdf.

3. van de Donk NW, et al. Treatment of relapsed and refractory multiple myeloma in the era of novel agents. Cancer Treat Rev. 2011;37(4): 266-283.

4. Orlowski M, Wilk S. Catalytic activities of the $20 \mathrm{~S}$ proteasome, a multicatalytic proteinase complex. Arch Biochem Biophys. 2000;383(1):1-16.

5. Rock KL, et al. Inhibitors of the proteasome block the degradation of most cell proteins and the generation of peptides presented on MHC class I molecules. Cell. 1994;78(5):761-771.

6. Kuhn DJ, et al. Potent activity of carfilzomib, a novel, irreversible inhibitor of the ubiquitin-proteasome pathway, against preclinical models of multiple myeloma. Blood. 2007;110(9):3281-3290.

7. Meng L, et al. Epoxomicin, a potent and selective proteasome inhibitor, exhibits in vivo antiinflammatory activity. Proc Natl Acad Sci U S A. 1999;96(18):10403-10408.

8. Parlati F, LS, Aujay M, et al. Carfilzomib: a selective inhibitor of the chymotrypsin-like activity of the constitutive proteasome and immunoproteasome has anti-tumor activity on multiple myeloma, lymphoma, and leukemia cells with minimal effects on normal cells. Haematologica. 94( Suppl 2).

9. Demo SD, et al. Antitumor activity of PR-171, a novel irreversible inhibitor of the proteasome. Cancer Res. 2007;67(13): 6383-6391.

10. Suzuki E, et al. Bortezomib-Resistant Cell Lines Have Increased Proteasome Levels but Remain Sensitive to Carfilzomib. ASH Annual Meeting Abstracts. 2009;114(22):2852.

11. Ivancsits D, et al. The Proteasome Inhibitor PR-171 Inhibits Cell Growth, Induces Apoptosis, and Overcomes De Novo and Acquired Drug Resistance in Human Multiple Myeloma Cells. ASH Annual Meeting Abstracts. 2005;106(11):1575.

12. Alsina $\mathrm{M}$, et al. A phase I single-agent study of twice-weekly consecutive-day dosing of the proteasome inhibitor carfilzomib in patients with relapsed or refractory multiple myeloma or lymphoma. Clin Cancer Res. 2012;18(17):4830-4840. 
13. O'Connor O A, et al. A phase 1 dose escalation study of the safety and pharmacokinetics of the novel proteasome inhibitor carfilzomib (PR-171) in patients with hematologic malignancies. Clin Cancer Res. 2009;15(22):7085-7091.

14. Kyprolis (carfilzomib) for Injection; Available from: http://www. accessdata.fda.gov/drugsatfda_docs/label/2012/202714lbl.pdf.

15. Siegel DS, et al. A phase 2 study of single-agent carfilzomib (PX171-003-A1) in patients with relapsed and refractory multiple myeloma. 2012;120:2817-2825.

16. Papadopoulos KP, et al. Phase I Study of 30-Minute Infusion of Carfilzomib As Single Agent or in Combination With Low-Dose Dexamethasone in Patients With Relapsed and/or Refractory Multiple Myeloma. J Clin Oncol. 2014.

17. Vij R, et al. An open-label, single-arm, phase 2 (PX-171-004) study of single-agent carfilzomib in bortezomib-naive patients with relapsed and/or refractory multiple myeloma. Blood. 2012;119(24): 5661-5670.

18. Amgen Announces Top-Line Results From Phase 3 Focus Trial Of Kyprolis ${ }^{\circledR}$ In Patients With Relapsed And Advanced Refractory Multiple Myeloma. August 12, 2014]; Available from: http://www.amgen. com.

19. Klein L, et al. A Phase 1, Dose-Escalation Study (CHAMPION-1) Investigating Weekly Carfilzomib In Combination With Dexamethasone For Patients With Relapsed Or Refractory Multiple Myeloma. 2013;122:1934-1934.

20. Lendvai $\mathrm{N}$, et al. A phase 2 single-center study of carfilzomib $56 \mathrm{mg} / \mathrm{m}^{2}$ with or without low-dose dexamethasone in relapsed multiple myeloma. Blood. 2014;124(6):899-906.

21. Wang M, et al. Phase 2 dose-expansion study (PX-171-006) of carfilzomib, lenalidomide, and low-dose dexamethasone in relapsed or progressive multiple myeloma. Blood. 2013;122(18):3122-3128.

22. Niesvizky R, et al. Phase Ib dose-escalation study (PX-171-006) of carfilzomib, lenalidomide, and low-dose dexamethasone in relapsed or progressive multiple myeloma. Clin Cancer Res. 2013;19(8):2248-2256.

23. Stadtmauer EA, et al. Phase I/II Dose Expansion Of a Multi-Center Trial Of Carfilzomib and Pomalidomide With Dexamethasone (CarPom-d) In Patients With Relapsed/Refractory Multiple Myeloma. 2013;122:690-690.

24. Feng L, et al. Phase 1 Study Of The Novel Kinesin Spindle Protein Inhibitor ARRY-520 + Carfilzomib (Car) In Patients With Relapsed and/ Or Refractory Multiple Myeloma (RRMM). 2013;122:1982-1982.

25. Amgen. Amgen Announces Phase 3 ASPIRE Trial of Kyprolis In Patients With Relapsed Multiple Myeloma Met Primary Endpoint. August 4, 2014; Available from: http://www.amgen.com.

26. Jakubowiak AJ, et al. Treatment outcome with the combination of carfilzomib, lenalidomide, and low-dose dexamethasone (CRd) for newly diagnosed multiple myeloma (NDMM) after extended follow-up. ASCO Meeting Abstracts. 2013;31(15_suppl):8543.

27. Allan JN, et al. Car-Bird [Carfilzomib, Clarithromycin(Biaxin(R)), Lenalidomide/(Revlimid(R)), Dexamethasone) For Newly-Diagnosed Multiple Myeloma. 2013;122:3216-3216.

28. Zingone A, et al. Phase II Clinical and Correlative Study Of Carfilzomib, Lenalidomide, and Dexamethasone Followed By Lenalidomide Extended Dosing (CRD-R) Induces High Rates Of MRD Negativity In Newly Diagnosed Multiple Myeloma (MM) Patients. 2013;122:538-538.

29. Asselberg-Hacker E, et al. Dose Escalation Phase 2 Trial Of Carfilzomib Combined With Thalidomide and Low-Dose Dexamethason In Newly Diagnosed, Transplant Eligible Patients With Multiple Myeloma. A Trial Of The European Myeloma Network. 2013;122:688-688.

30. Reeder CB, et al. A Phase I/II Trial Of Cyclophosphamide, Carfilzomib, Thalidomide and Dexamethasone (CYCLONE) In Patients With Newly Diagnosed Multiple Myeloma: Final Results Of MTD Expansion Cohort. 2013;122:3179-3179.
31. Mailankody S, et al. Clinical and Correlative Pilot Study Of Carfilzomib, Lenalidomide, and Dexamethasone Followed By Lenalidomide Extended Dosing (CRd - R) In High Risk Smoldering Multiple Myeloma Patients. 2013;122:1939-1939.

32. Blade J, et al. Renal failure in multiple myeloma: presenting features and predictors of outcome in 94 patients from a single institution. Arch Intern Med. 1998;158(17):1889-1893.

33. Badros AZ, et al. Carfilzomib in multiple myeloma patients with renal impairment: pharmacokinetics and safety. Leukemia. 2013;27(8):1707-1714

34. Wisloff $F$, et al. Has the incidence of multiple myeloma in old age been underestimated? The myeloma project of health region I in Norway. I. Eur J Haematol. 1991;47(5):333-337.

35. Jagannath $\mathrm{S}$, et al. An open-label single-arm pilot phase II study (PX171-003-A0) of low-dose, single-agent carfilzomib in patients with relapsed and refractory multiple myeloma. Clin Lymphoma Myeloma Leuk. 2012.;12(5):310-318.

36. Bringhen S, et al. Carfilzomib, cyclophosphamide, and dexamethasone in patients with newly diagnosed multiple myeloma: a multicenter, phase 2 study. Blood. 2014;124(1):63-69.

37. Touzeau C, et al. Effect of CMP, carfilzomib (CFZ) plus melphalanprednisone (MP), on response rates in elderly patients (pts) with newly diagnosed multiple myeloma (NDMM): Results of a phase (Ph) I/II trial. ASCO Meeting Abstracts. 2013;31(15_suppl):8513.

38. Munshi NC, et al. Consensus recommendations for risk stratification in multiple myeloma: report of the International Myeloma Workshop Consensus Panel 2. Blood. 2011;117(18):4696-4700.

39. Sawyer JR. The prognostic significance of cytogenetics and molecular profiling in multiple myeloma. Cancer Genet. 2011;204(1):3-12.

40. Morgan GJ, Walker BA, FE Davies. The genetic architecture of multiple myeloma. Nat Rev Cancer. 2012;12(5):335-348.

41. Kyle RA, Rajkumar SV. Criteria for diagnosis, staging, risk stratification and response assessment of multiple myeloma. Leukemia. 2009;23(1):3-9.

42. Neben K, et al. Administration of bortezomib before and after autologous stem cell transplantation improves outcome in multiple myeloma patients with deletion 17p. Blood. 2012;119(4):940-948.

43. Avet-Loiseau H, et al. Bortezomib plus dexamethasone induction improves outcome of patients with $\mathrm{t}(4 ; 14)$ myeloma but not outcome of patients with del(17p). J Clin Oncol. 2010;28(30): 4630-4634.

44. Jagannath $\mathrm{S}$, et al. Bortezomib appears to overcome the poor prognosis conferred by chromosome 13 deletion in phase 2 and 3 trials. Leukemia. 2007;21(1):151-157.

45. Jakubowiak AJ, et al. Treatment outcomes in patients with relapsed and refractory multiple myeloma and high-risk cytogenetics receiving single-agent carfilzomib in the PX-171-003-A1 study. Leukemia. 2013;27(12):2351-2356.

46. Siegel $\mathrm{D}$, et al. Integrated safety profile of single-agent carfilzomib: experience from 526 patients enrolled in 4 phase II clinical studies. Haematologica. 2013;98(11):1753-1761.

47. Onyx Pharmaceuticals. ClinicalTrials.gov. Phase 3 Study with Carfilzomib and Dexamethasone versus Velcade and Dexamethasone for Relapsed Multiple Myeloma Patients (ENDEAVOR). Available from: http://clinicaltrials.gov/show/NCT01568866. NLM identifier: NCT01568866. Accessed: August 10, 2014.

48. Onyx Pharmaceuticals. ClinicalTrials.gov. Phase 3 Study of Carfilzomib, Melphalan, Prednisone vs Bortezomib, Melphalan, Prednisone in Newly Diagnosed Multiple Myeloma (CLARION). Available from: http:// clinicaltrials.gov/show/NCT01818752. NLM identifier: NCT01818752. Accessed: August 10, 2014.

49. Columbia University. ClinicalTrials.gov. Carfilzomib With Bendamustine and Dexamethasone in Multiple Myeloma. Available from: http://clinicaltrials.gov/ct2/show/NCT02002598. NLM identifier: NCT02002598. Accessed: August 10, 2014. 
50. Medical University of South Carolina. ClinicalTrials.gov. Carfilzomib + High Dose Melphalan as Preparative Regimen for Autologous Hematopoietic Stem Cell Transplantation. Available from: http://clinicaltrials.gov/ ct2/show/NCT01690143. NLM identifier: NCT01690143. Accessed: August 10, 2014.

51. University of Chicago. ClinicalTrials.gov. Carfilzomib, Lenalidomide, and Dexamethasone Before and After Stem Cell Transplant in Treating Patients With Newly Diagnosed Multiple Myeloma. Available from: http:/clinicaltrials.gov/ct2/show/NCT01816971. NLM identifier: NCT01816971. Accessed: August 10, 2014.

52. National Cancer Institute. ClinicalTrials.gov. Carfilzomib, Lenalidomide, and Dexamethasone for Smoldering Multiple Myeloma. Available from: http:/clinicaltrials.gov/show/NCT01572480. NLM identifier: NCT01572480. Accessed: August 10, 2014.

53. Onyx Pharmaceuticals. ClinicalTrials.gov. Study of High Dose Carfilzomib in Multiple Myeloma Patients Who Have Progressed On Standard Dose Carfilzomib. Available from: http:/clinicaltrials.gov/ct2/show/ NCT01775553. NLM identifier: NCT01775553. Accessed: August 10, 2014.

54. Pharmacyclics. ClinicalTrials.gov. Study of the Bruton's Tyrosine Kinase Inhibitor in Combination With Carfilzomib (Kyprolis ${ }^{\mathrm{TM}}$ ), in Subjects With Relapsed or Relapsed and Refractory Multiple Myeloma. Available from: http://clinicaltrials.gov/ct2/show/NCT01962792. NLM identifier: NCT01962792. Accessed: August 10, 2014.

55. M.D. Anderson Cancer Center. ClinicalTrials.gov. Arry-520 + Carfilzomib for Multiple Myeloma (MM). Available from: http:// clinicaltrials.gov/ct2/show/NCT01372540. NLM identifier: NCT01372540. Accessed: August 10, 2014

56. Southwest Oncology Group. ClinicalTrials.gov. S1304, Testing Two Doses of Carfilzomib With Dexamethasone for Relapsed or Refractory Myeloma. Available from: http://clinicaltrials.gov/ct2/show/ NCT01903811. NLM identifier: NCT01903811. Accessed: August 10, 2014.
57. Emory University. ClinicalTrials.gov. A Phase I Study of the Combination of Panobinostat and Carfilzomib in Patients With Relapsed and/or Refractory Multiple Myeloma. Available from: http://clinicaltrials.gov/ ct2/show/NCT01549431. NLM identifier: NCT01549431. Accessed: August 10, 2014.

58. University of Chicago. ClinicalTrials.gov. Carfilzomib, Pomalidomide, and Dexamethasone in Treating Patients With Relapsed or Refractory Multiple Myeloma. Available from: http:/clinicaltrials.gov/ct2/show/ NCT01665794. NLM identifier: NCT01665794. Accessed: August 10, 2014.

59. Hackensack University Medical Center. ClinicalTrials.gov. A Study of Carfilzomib, Lenalidomide, Vorinostat, and Dexamethasone in Relapsed and/or Refractory Multiple Myeloma (QUAD). Available from: http:/clinicaltrials.gov/ct2/show/NCT01297764. NLM identifier: NCT01297764. Accessed: August 10, 2014.

60. Washington University School of Medicine. ClinicalTrials.gov. Carfilzomib, Pegylated Liposomal Doxorubicin Hydrochloride, and Dexamethasone in Treating Patients With Relapsed or Refractory Multiple Myeloma. Available from: http://clinicaltrials.gov/ct2/show/ NCT01246063. NLM identifier: NCT01246063. Accessed: August 10, 2014.

61. Oncotherapeutics. ClinicalTrials.gov. Safety, Efficacy, and Pharmacodynamics of a 60-Minute Infusion of Carfilzomib for Progressive Multiple Myeloma. Available from: http://clinicaltrials.gov/ct2/show/ NCT01792102. NLM identifier: NCT01792102. Accessed: August 10, 2014.

62. M.D. Anderson Cancer Center. ClinicalTrials.gov. Carfilzomib/SAHA Combined With High-Dose Gemcitabine/Busulfan/Melphalan With Autologous Stem Cell Transplant in Myeloma. Available from: http:// clinicaltrials.gov/show/NCT02114502. NLM identifier: NCT02114502. Accessed: August 10, 2014
Pharmacogenomics and Personalized Medicine

\section{Publish your work in this journal}

Pharmacogenomics and Personalized Medicine is an international, peerreviewed, open access journal characterizing the influence of genotype on pharmacology leading to the development of personalized treatment programs and individualized drug selection for improved safety, efficacy and sustainability. This journal is indexed on the American Chemical

\section{Dovepress}

Society's Chemical Abstracts Service (CAS). The manuscript management system is completely online and includes a very quick and fair peer-review system, which is all easy to use. Visit http://www.dovepress. com/testimonials.php to read real quotes from published authors. 